\title{
Large doses of intravenous Rh (D) immunoglobulin lead to sustained elevations in Rh antibody titers
}

\author{
Vani C Movva, MBBS, ${ }^{1}$ Asha Rijhsinghani, $\mathrm{MD}^{1}$ \\ Keywords: Alloimmunization, mismatched blood transfusion, Rh immunoglobulin, anti Rh- D titers, \\ exchange transfusion
}

\begin{abstract}
Rhesus (Rh) alloimmunization is potentially devastating for reproductive health. Rarely, RhD mismatched blood may be transfused accidentally, increasing the risks of $R h D$ sensitization. Prevention of $R h$ sensitization is important especially in women of reproductive age group. Treatment with intravenous Rhlg is potentially safe and also the effective means of preventing $R h$ alloimmunization in cases of $R h$ mismatched blood transfusion. We report a case that illustrates the significantly high $R h D$ antibody titers following treatment with intravenous Rh immunoglobulin for prevention of alloimmunization. An 18yo Rh-negative G0 had mistakenly received four units of Rh-positive blood. To avoid future pregnancy related complications, she received monotherapy with 13,200 $\mu \mathrm{g}$ total of intravenous $R h$ immunoglobulin (Rhlg). The treatment was well tolerated; however the RhD antibody titers reached a peak of 1:512. The high levels of $R h D$ antibody titers can have grave implications if the patient is pregnant.
\end{abstract}

${ }^{1}$ Perinatal Care Clinics, Department of Obstetrics and Gynecology, University of lowa Hospitals and Clinics lowa City, lowa.

\section{Introduction}

Alloimmunization in most obstetric cases occurs after transplacental passage of fetal blood into the maternal system during pregnancy-related causes or events. Rarely can it occur following $\mathrm{RhD}$ mismatched blood transfusion. The incidence of mismatched transfusion is quoted to be approximately 1 in 100,000 US population. In 2008, a total of 165 incompatible blood transfusions were reported in the United States community hospitals. ${ }^{1}$

Prevention of alloimmunization in women of reproductive age is of utmost importance to avoid future pregnancy complications. In cases of RhD mismatched blood transfusion, the suggested treatments include red blood cell exchange transfusion and administration of intravenous (IV) rhesus immune globulin (Rhlg) within 72 hours. Described here is a case of $\mathrm{RhD}$

Please cite this paper as: Movva VC, Rijhsinghani A. Large doses of intravenous Rh (D) immunoglobulin lead to sustained elevations in Rh antibody titers. Proc Obstet Gynecol. 2014;4(2): Article 6 [ 6 p.]. Available from: http://ir.uiowa.edu/pog/. Free full text article.

Corresponding author: Asha Rijhsinghani, MD, Department of Obstetrics and Gynecology, University of lowa Hospitals and Clinics, 200 Hawkins Drive, lowa City, lowa 52242-1080, Telephone: (319) 356-1945, FAX: (319) 353-6759, asharijhsinghani@uiowa.edu.

Financial Disclosure: The authors report no conflict of interest.

Copyright: (C) 2014 Rijhsinghani, et al. This is an open-access article distributed under the terms of the Creative Commons Attribution License, which permits unrestricted use, distribution, and reproduction in any medium, provided the original author and source are credited. 
mismatched transfusion and treatment with only intravenous rhesus immune globulin (WinRho SD, NABI, Boca Raton, FL). The treatment was associated with significant, but transient elevations in Rh anti $D$ antibody titers.

\section{Case Report}

An 18-year-old nulligravid woman was involved in a high-speed motor vehicle accident and was found to be unstable with a blood pressure of $76 / 38 \mathrm{~mm} \mathrm{Hg}$. The initial work-up revealed extensive internal injuries and several fractures, as well as a small subdural hematoma. The patient emergently received four units of RhD positive blood. Following the transfusion, it was realized that the patient was RhD negative.

The implications of $\mathrm{RhD}$ incompatibility for future pregnancies were discussed with the patient. Intramuscular (IM) Rhlg was determined to be impractical because of the large volumes needed. The option of red blood cell exchange transfusion was considered, though with a delay, due to concerns of increasing the intracranial pressure in the presence of a subdural hematoma. The patient and her family decided against the exchange transfusion for fear of complications. Due to the critical condition of the patient, the obstetrical team was consulted only fourteen days after admission regarding the implications of $\mathrm{RhD}$ sensitization in a woman of reproductive age group. The patient was counseled about obstetrical risks associated with $\mathrm{RhD}$ alloimmunization, and was offered treatment with intravenous (IV) Rhlg. She decided in favor of this treatment.
The dose of Rhlg was calculated based on the amount of RhD mismatched blood transfused. The patient had received 1200 cc of blood. With a $55 \%$ hematocrit, that is equivalent to an exposure of 660 ccs of Rh positive red blood cells. The total dose of IV Rhlg (dosed at 90 IU of IV Rhlg per $1 \mathrm{~mL}$ of mismatched PRBCs) was calculated at 59,400 IU for our patient. Since the maximum daily dose of the IV Rhlg advised is 3000 IU every 8 hours, we gave the total dose over a seven day period to ensure adequate treatment. $\mathrm{RhD}$ antibody titers were obtained daily during this treatment, and repeated monthly after discharge. The patient tolerated the treatment well and did not show any signs of overt jaundice or renal complications, and did not require any blood transfusion. The anti RhD antibody titers rose to a peak of 1:512, dropping to $1: 8$ at the end of the 3rd month. Even with the high RhD antibody titers, serum bilirubin levels remained below 2mg/ dl. [Figure 1] Follow up titers were not available after the 3rd month.

\section{Discussion}

The reported rate of mismatched blood transfusion is approximately 1 per 12,000 units $^{2}$ and the complications of which are multiple. Factors such as amount of mismatched blood received and immune status contribute to the likelihood of $\mathrm{RhD}$ alloimmunization, which is approximately $80 \%$ after transfusion with $500 \mathrm{cc}$ of $\mathrm{RhD}$ mismatched blood. $^{3}$ After the first exposure to RhD antigen, antibodies may not be detectable for at least 4 weeks. $^{4}$ 
In a mismatched blood transfusion, a red blood cell exchange transfusion or Rhlg injection has been used successfully for prevention of $\mathrm{RhD}$ sensitization. The mechanism by which the Rhlg inhibits sensitization is hypothesized to be by antigen clearance or by alteration of antigen processing and presenting. ${ }^{5}$



Figure 1: Anti $D$ antibody titer and total bilirubin levels following administration of intravenous Rhlg 
When the volume of the inappropriate blood transfused is large, a combination of exchange transfusion and IV Rhlg has been proposed. ${ }^{6}$ Nester, et al described two cases where $\mathrm{RhD}$ negative women received RhD positive blood and were successfully treated with the combination therapy. ${ }^{6}$ Both women had negative RhD antibody titers six months after the treatment.

Usage of Rhlg alone, has been reported with success as well as failure which is probably dependent on the dosing of the Rhlg. ${ }^{7,8}$ Blood volume as low as $0.1 \mathrm{ml}$ can lead to $\mathrm{Rh}$ sensitization. ${ }^{9}$ Inadequacy in the dosing of the Rhlg could lead to failure of prophylaxis. In our case, even though the volume of the inappropriate blood transfused was large, we decided in favor of IV Rhlg monotherapy due to the 14 day delay in the treatment. The dose of IV Rhlg administered was large and calculated to cover the large volume of the RhD mismatched transfused blood, but the patient tolerated the treatment well.

In the previously reported case of treatment failure with Rhlg alone, the patient was given 1,650 $\mu \mathrm{g}$ Rhlg, for a single unit of $\mathrm{RhD}$ inappropriate blood transfusion. ${ }^{7}$ In that case the RhD antibody titers were reported to rise to: $1: 8,1: 512,1: 512$, and 1:64, on posttransfusion days $6,70,119$, and 369 respectively. The persistence of antibodies more than one year later was taken as evidence of treatment failure, by the authors. In our case, following treatment with IV Rhlg, the antibody titers rose significantly but the rise did not persist. By post-transfusion day 91, the RhD antibody titers had fallen from 1:512 to 1:8. Though we feel reassured with the drop in the titers, we cannot categorically comment on the long-term success of the management since the patient was lost to follow up.

In RhD sensitized pregnancies, RhD antibody titer between $1: 8$ and $1: 32$ is defined as the critical titer, above which the $\mathrm{RhD}$ positive fetus is at a significant risk for fetal hydrops. ${ }^{10}$ The peak titer achieved in our case was 1:512 following treatment with the IV Rhlg, and the higher titers persisted over a period of 2 months. Though our patient was not pregnant, the elevation in the $\mathrm{RhD}$ antibody titers has implications in a pregnant woman carrying an $\mathrm{RhD}$ positive fetus.

In recent times, IV Rhlg has also been used successfully in the treatment of immune thrombocytopenic purpura (ITP) during pregnancy, the exact mechanism of which is not well understood. ${ }^{11,12} \mathrm{Rhlg}$ demonstrates the ability to block the reticuloendothelial system from clearing antibody-coated cells, and to also modulate the immune system. ${ }^{12}$ Management of conditions such as mismatched blood transfusion and ITP with large doses of Rhlg can lead to $\mathrm{RhD}$ antibody titers rising beyond the critical levels and can be potentially harmful to the fetus if the patient is pregnant.

\section{Conclusion}

It appears that IV Rhlg alone is a safe and potentially effective treatment for prevention of alloimmunization following $\mathrm{RhD}$ mismatched blood transfusion, even when the volume is large. In cases of RhD mismatched blood transfusions, if the treatment is delayed beyond 14 days, IV Rhlg should still be considered as long as the patient has a 
negative pre-treatment RhD antibody titer. However, due to the sharp rise in the RhD antibody titers following treatment, caution should be exercised when considering IV RhIg for treatment in pregnant women.

Acknowledgments: The authors would like to thank S Sung for data collection and Dr. MK Santillan for the graph.

\section{References}

1. U.S. Department of Health and Human Services. Agency for Healthcare Research and Quality. National Quality Measures Clearinghouse. Measure summary. Transfusion reaction (arealevel): rate per 100,000 population [Internet]. Washington 2012 March [cited 2014 July 1]. Available from: http://www.qualitymeasures.ahrq.gov/co ntent.aspx?id=38536.

2. Linden JV. Errors in transfusion medicine. Scope of the problem. Arch Pathol Lab Med. 1999 Jul;123(7):563-5. PubMed PMID: 10388907.

3. Pollack W, Ascari WQ, Crispen JF, O'Connor RR, Ho TY. Studies on Rh prophylaxis. II. Rh immune prophylaxis after transfusion with Rh-positive blood. Transfusion. 1971 Nov-Dec;11(6):340-4. http://dx.doi.org/10.1111/j.1537-

2995.1971.tb04425.x. PubMed PMID: 5002766.

4. Urbaniak SJ. Alloimmunity to $\mathrm{RhD}$ in humans. Transfus Clin Biol. 2006 MarApr;13(1-2):19-22. Epub 2006 Mar 30. DOI: $\quad$ 10.1016/j.tracli.2006.02.020. PubMed PMID: 16574456.

5. Brinc D, Lazarus AH. Mechanisms of anti-D action in the prevention of hemolytic disease of the fetus and newborn. Hematology Am Soc Hematol Educ Program. 2009:185-91. doi: 10.1182/asheducation-2009.1.185.

PubMed PMID: 20008198.
6. Nester TA, Rumsey DM, Howell CC, Gilligan DM, Drachman JG, Maier RV, Kyles DM, Matthews DC, Pendergrass TW. Prevention of immunization to D+ red blood cells with red blood cell exchange and intravenous $\mathrm{Rh}$ immune globulin. Transfusion. 2004 Dec;44(12):1720-3.

http://dx.doi.org/10.1111/j.0041-

1132.2004.04161.x. PubMed PMID: 15584986.

7. Ahrens J, Heuft HG, Goudeva L, Przemeck M. Rhesus immune globulin fails to prevent immunization after rhesus incompatible blood transfusion. Transfus Apher Sci. 2007 Apr;36(2):139-42. Epub 2007 Mar 23. DOI: $\quad$ 10.1016/j.transci.2006.10.009. PubMed PMID: 17368103.

8. Werch JB. Prevention of Rh sensitization in the context of trauma: two case reports. J Clin Apher. 2010;25(2):70-3. doi: 10.1002/jca.20225. PubMed PMID:20027640.

9. Woodrow JC, Finn R. Transplacental haemorrhage. $\mathrm{Br} \mathrm{J}$ Haematol. 1966 May;12(3):297-309.

http://dx.doi.org/10.1111/j.1365-

2141.1966.tb05636.x. PubMed PMID: 4956849.

10. American College of Obstetricians and Gynecologists. ACOG Practice Bulletin No. 75 : Management of alloimmunization during pregnancy. Obstet Gynecol. 2006 Aug;108(2):45764. http://dx.doi.org/10.1097/00006250200608000-00044. PubMed PMID: 16880320.

11. Sieunarine K, Shapiro S, Al Obaidi M, Girling J. Intravenous anti-D immunoglobulin in the treatment of resistant immune thrombocytopenic purpura in pregnancy. BJOG. 2007 Apr;114(4):505-7. Epub 2007 Feb 19. http://dx.doi.org/10.1111/j.1471-

0528.2007.01234.x. PubMed PMID: 17309547. 
12. Lazarus $A H$, Crow AR. Mechanism of action of IVIG and anti-D in ITP. Transfus Apher Sci. 2003 Jun;28(3):249-55. DOI: 10.1016/S14730502(03)00043-0. PubMed PMID: 12725951. 